The INL is a

U.S. Department of Energy

National Laboratory

operated by

Battelle Energy Alliance

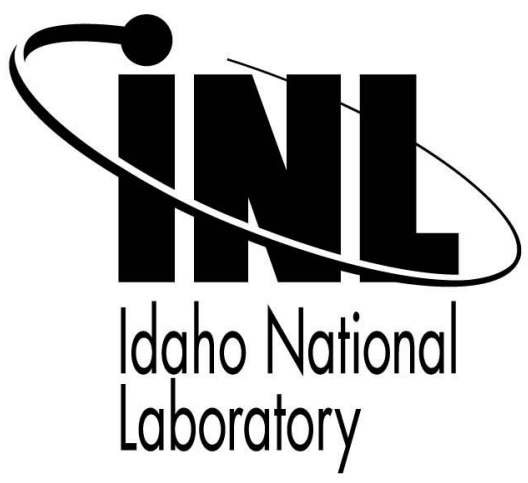

INL/CON-07-12500

PREPRINT

\title{
Catalytic Activity of Supported Metal Particles for Sulfuric Acid Decomposition Reaction
}

\section{$234^{\text {th }}$ American Chemical Society Meeting}

\author{
Sergey N. Rashkeev \\ Daniel M. Ginosar \\ Lucia M. Petkovic \\ Helen H. Farrell
}

\section{August 2007}

This is a preprint of a paper intended for publication in a journal or proceedings. Since changes may be made before publication, this preprint should not be cited or reproduced without permission of the author. This document was prepared as an account of work sponsored by an agency of the United States Government. Neither the United States Government nor any agency thereof, or any of their employees, makes any warranty, expressed or implied, or assumes any legal liability or responsibility for any third party's use, or the results of such use, of any information, apparatus, product or process disclosed in this report, or represents that its use by such third party would not infringe privately owned rights. The views expressed in this paper are not necessarily those of the United States Government or the sponsoring agency. 


\title{
Catalytic activity of supported metal particies for sulfuric acid decomposition reaction
}

\author{
Sergey N. Rashkeev ${ }^{\mathrm{a}, *}$, Daniel M. Ginosar ${ }^{\mathrm{b}}$, Lucia M. Petkovic ${ }^{\mathrm{b}}$, Helen H. Farrell ${ }^{\mathrm{c}}$ \\ ${ }^{a}$ Center for Advanced Modeling and Simulation, Idaho National Laboratory, Idaho Falls, ID 83415, United States \\ b Chemical Sciences Division, Idaho National Laboratory, Idaho Falis, ID 83415, United States \\ 'Materials Science Division, Idaho National Laboratory, Idaho Falls, ID 83415, United States
}

Keywords:

Thermochemical water splitting

Sulfur-based cycles

Sulfuric acid decomposition

Hydrogen production

Nanoclusters

Density-functional theory

\begin{abstract}
A B S T R ACT
Production of hydrogen by splitting of water in the thermochemical sulfur-based cycles that employs the catalytic decomposition of sulfuric acid into $\mathrm{SO}_{2}$ and $\mathrm{O}_{2}$ is of considerable interest. However, all of the known catalytic systems studied to date that consist of metal particles on oxide substrates deactivate with time on stream. To develop an understanding of the factors that are responsible for catalyst activity, we investigate the fresh activity of several platinum group metals (PGM) catalysts, including Pd, Pt, Rh, Ir. and Ru supported on titania at $850^{\circ} \mathrm{C}$ and perform an extensive theoretical study (density-functionaltheory-based first-principles calculations and computer simulations) of the activity of the PGM nanoparticles of different size and shape positioned on $\mathrm{TiO}_{2}$ (rutile and anatase) and $\mathrm{Al}_{2} \mathrm{O}_{3}(\gamma$ - and $\eta$ alumina) surfaces. The activity and deactivation of the catalytic systems are defined by (i) the energy barrier for the detachment of $\mathrm{O}$ atoms from the $\mathrm{SO}_{n}(n=1,2,3)$ species, and (ii) the removal rate of the products of the sulfuric acid decomposition (atomic $\mathrm{O}, \mathrm{S}$, and the $\mathrm{SO}_{n}$ species) from metal nanoparticles. We show that these two nanoscale features collectively result in the observed experimental behavior. The removal rate of the reaction products is always lower than the $\mathrm{SO}_{n}$ decomposition rates. The relation between these two rates explains why the "softer" PGM nanoparticles (Pd and Pt) exhibit the highest initial catalytic activity.
\end{abstract}

\section{Introduction}

Thermochemical cycles produce hydrogen through a series of chemical reactions where the net result is the production of hydrogen and oxygen from water at much lower temperatures than direct thermal decomposition [1]. The catalytic chemicals within this process are recycled, and the heat to drive the reactions must be provided by a primary energy source. When the primary energy driver is nuclear or solar heat, hydrogen can be generated without producing green-house gasses, and can provide independence from dwindling supplies of fossil fueis.

The sulfur-based family of thermochemical cycles, including the Sulfur-lodine cycle [2], the Hybrid Sulfur cycle [3], and the Sulfur-Bromine Hybrid cycle [4], incorporate the sulfuric acid decomposition reaction. This reaction takes place in two steps, a non-catalytic thermal decomposition of the acid to form gaseous $\mathrm{SO}_{3}$ and $\mathrm{H}_{2} \mathrm{O}$ at temperatures above $350^{\circ} \mathrm{C}$, followed by catalytic decomposition of the $\mathrm{SO}_{3}$ at temperatures above $750{ }^{\circ} \mathrm{C}$ to produce

\footnotetext{
Corresponding author. Tel.: +1 2085269402

E-mail address: Sergey.Rashkeev@inl.gov (S.N. Rashkeev).
}

the $\mathrm{SO}_{2}$ and $\mathrm{O}_{2}$ products. The production of $\mathrm{O}_{2}$ represents half of the water splitting reaction and is a product from the cycle. The production of hydrogen is accomplished through a second reaction specific to the individual cycle. In the Sulfur-Iodine cycle, hydrogen is generated by the decomposition of $\mathrm{HI}$ which also produces $\mathrm{I}_{2}$ that is recycled to the main reaction.

At sulfuric acid decomposition operating temperatures between 750 and $900{ }^{\circ} \mathrm{C}$, the reduction of $\mathrm{SO}_{3}$ requires a catalyst that may work at extremely high temperatures and under exposure to chemically aggressive environments (steam, oxygen, acid vapor, etc.) that severely damage and destroy most catalysts within a short time. A number of catalysts for sulfuric acid decomposition were previously explored, and activities of over 30 catalysts have been reported [5-8]. Those studies reported that most metal oxides had low activity due to sulfate formation [5-7], which is more significant at lower reaction temperatures. In addition, some catalysts were found to fail due to formation of volatile acid salts [5], support poisoning [5], and catalyst attrition [8]. Our previous experiments show that platinum supported on titania (rutile) was more stable than on several other supports [9]; however, even on titania, the Pt sites deactivate over long periods of time due to sintering, vaporization, and oxidation $[9,10]$. 
Therefore, it is of utmost importance to make catalysts more effective by enhancing their stability and reducing their cost.

In this work, we present the fresh, or initial, activity of platinum group metals (PGM), including Pd, Pt, Rh, Ir, and Ru supported on titania at $850{ }^{\circ} \mathrm{C}$ and perform an extensive theoretical study of the activity of $\mathrm{Pd}, \mathrm{Pt}, \mathrm{Rh}, \mathrm{Ir}, \mathrm{Ru}$, and Os nanoparticles positioned on titania (rutile and anatase) and alumina ( $\gamma$ - and $\eta$-alumina) surfaces in the catalytic sulfuric acid decomposition. The choice of the considered transition metals (PGMs) was inspired by their high melting temperatures--we expected that nanoparticles of these metals will be more stable at high temperatures. Although Pd has a lower melting temperature, it was included to make this study more comprehensive. To develop understanding of the factors that are responsible for the catalyst activity and the deactivation, we perform first-principles calculations for metal nanoparticles of different size and structure, and investigate the interaction between these particles (accommodated at oxide substrates) and the $\mathrm{SO}_{n}(n=1,2,3)$ species (the products of the sulfuric acid decomposition). We show that the behavior of the catalytic particles is defined by several nanoscale features and explain the reasons for the deactivation of the catalysts with time on stream.

\section{Experiment, theory, and simulations}

\subsection{Experimental method}

PGMs, including $\mathrm{Pd}, \mathrm{Pt}, \mathrm{Rh}, \mathrm{Ir}$, and Ru, supported on titania (rutile) were purchased from Johnson Matthey with nominal metal loadings of $0.1 \mathrm{wt} \%$. An osmium catalyst could not be obtained. Reaction tests were run at $850^{\circ} \mathrm{C}$ using $100 \mathrm{mg}$ of catalyst and a sulfuric acid (95.6wt\%, Fischer Scientific) flow rate of $24 \mathrm{~g} / \mathrm{h}$ resulting in a weight hourly space velocity (WHSV) of about $230 \mathrm{~g}$ $\mathrm{H}_{2} \mathrm{SO}_{4} / \mathrm{h} / \mathrm{g}$ catalyst $\left(\mathrm{h}^{-1}\right)$. Catalyst samples were used as coarse powders sieved between 40 and 60 mesh. The rate, temperature, catalyst loading and particle size were chosen to minimize thermodynamic and heat and mass transport limitations. It should be noted that the actual size of the PGM nanoparticles was not determined.

The experiments were conducted in a high-temperature continuous-flow catalyst testing system built of Teflon tubing and glass that was described previously [11]. The system employs a $4 \mathrm{~mm}$ ID quartz reactor placed in an electrically heated tube furnace. The system includes two top loading balances for feed and unreacted sulfuric acid, a pulse-free peristaltic feed pump, a mass flow controller for a reference gas stream, and an on-line gas chromatograph. The gas chromatograph (Agilent 3000A Micro GC) is equipped with both molecular sieve and PLOT Q columns and thermal conductivity detectors (TCD) for $\mathrm{SO}_{2}$ and $\mathrm{O}_{2}$ product quantification. The reactor effluent gas was sampled every 5 min.

The reaction experiments were also described previously [11]. Briefly, the reactor was heated from room temperature to $400{ }^{\circ} \mathrm{C}$ in $1 \mathrm{~h}$ in a flow of helium. The catalyst was then reduced at $400{ }^{\circ} \mathrm{C}$ in flowing hydrogen for $2 \mathrm{~h}$. The gas flow was switched back to helium and then the reactor was heated to the experiment temperature of $850^{\circ} \mathrm{C}$ over $2 \mathrm{~h}$. Once the system temperature stabilized, the flow of gas was terminated and liquid sulfuric acid was pumped to the reactor. Exiting the reactor, any unreacted acid was condensed and collected and the gaseous products $\left(\mathrm{SO}_{2}\right.$ and $\mathrm{O}_{2}$ ) flowed to the $\mathrm{GC}$ and then vented to a fume hood.

\subsection{Theory and simulations}

In order to investigate the role of different nanoparticle features in the sulfuric acid decomposition, we constructed a large ensemble of metal nanoparticles of different size and shape on $\mathrm{TiO}_{2}$ and $\mathrm{Al}_{2} \mathrm{O}_{3}$ substrates and optimized their geometries using density-functional

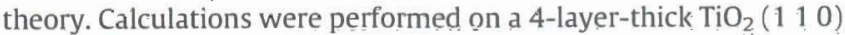
rutile slab and a 3-layer thick ( $\left.\begin{array}{lll}1 & 0 & 1\end{array}\right)$ anatase slab. Both slabs were terminated by non-polar surfaces. Slabs of $\gamma$ - and $\eta-\mathrm{Al}_{2} \mathrm{O}_{3}$ fiveatomic-layers-thick were cut from fully relaxed bulk structures [12] to expose the (110C) surface, which is energetically preferred [13]. Large, periodically repeated supercells were used $(13.0 \AA \times$ $12.4 \AA \times 23.5 \AA$ for rutile, $18.9 \AA \times 12.7 \AA \times 23.5 \AA$ for anatase, and $15.0 \AA \times 10.5 \AA \times 20.0 \AA$ for $\gamma$ - and $\eta$-aluminas). The vacuum layer between slabs was $>12 \AA$.

Small transition metal (TM) particles (below $1 \mathrm{~nm}$ in diameter) containing between 5 and 40 atoms on oxide slabs (Fig. 1a) were constructed as follows. First, we tried to find the anchoring site for a single transition metal atom at a given oxide surface. For the $\mathrm{TiO}_{2}$ (rutile and anatase) surfaces we used the defectless surfaces as well as the surfaces with oxygen vacancies. We found that for each of the considered transition metals (PGMs) the presence of oxygen vacancies is not as crucial for good adsorption as it is for gold [14].

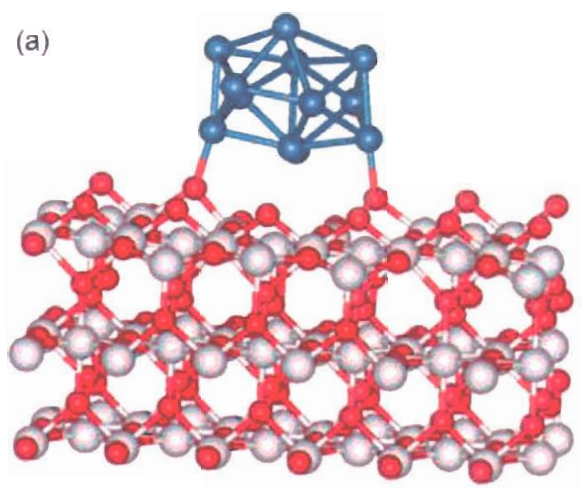

(b)

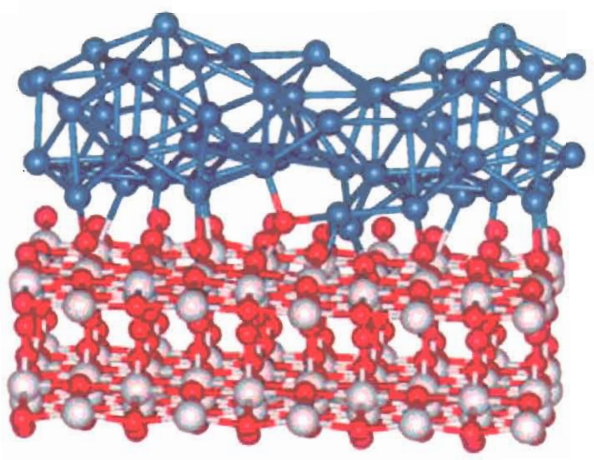

(c)

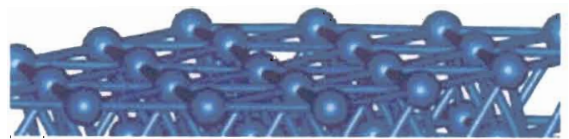

Fig. 1. Different types of the transition metal nanoparticles used for modeling and simulation of the sulfuric acid decomposition. Ti atoms are shown in light grey, $\mathrm{O}$ in red, TM in blue. (a) A relaxed configuration of 12-atoms Pt nanoparticle positioned on the $\mathrm{TiO}_{2}$ (rutile) surface on the top of an oxygen vacancy (an example of a TM nanoparticle with a diameter $\leq 1 \mathrm{~nm}$ ); (b) a relaxed quasi-one-dimensional (rodlike) Pt structure on the rutile surface (an example of a TM particle used for modeling of the processes on larger particles and on the particle-substrate boundaries); (c) a "bulk" Pt ( $\left.\begin{array}{lll}1 & 1 & 1\end{array}\right)$ surface (an example of the structure used for modeling of the processes on large, "quasi-flat" TM particles). (For interpretation of the references to color in this figure legend, the reader is referred to the web version of the article.) 
Nanoparticles with larger number of atoms were constructed by repeatedly adding a single metal atom to an existing particle and allowing the structure to relax until the quantum-mechanical force on each atom in the supercell became smaller than $0.02 \mathrm{eV} / \AA$. The supercells are sufficiently large so that even 40 -atom nanoparticles interact with their neighbors only weakly. In these calculations we did not perform a systematic search for the lowest energy structure. Instead, we found that it is more useful to construct an ensemble of nanoparticles with diverse local bonding and coordination numbers. Once we had an ensemble of stable nanoparticles for one transition metal (we started with Pt), all TM atoms of one sort were replaced by TM atoms of another sort, and all of the structures were relaxed again (typically, such a relaxation modifies only the interatomic distances in the particle while the site coordination remains unchanged).

For simulation of processes on larger nanoparticles, quasi-onedimensional periodic rod-like structures containing a well-defined boundary between the nanoparticle and the substrate were used. Our objective was to have stable structures with a well-defined "perimeter" between the nanoparticle and the surface where we could explore catalytic activity and particle stability (Fig. 1b). For modeling the processes on the surfaces of larger particles, we simply used surfaces of "bulk" transition metals (Fig. 1c). The bulk structure is face-centered cubic (fcc) for Pd, Pt, Rh, and Ir and hexagonal close-packed (hcp) for Ru and Os.

Although the particles constructed in such a way cannot be taken exactly of the size of metal nanoparticles used in real catalytic processes (some of these particles may contain thousands of atoms, and the first-principles calculations for so big systems are not affordable at the present), we have a possibility to model the structure and electronic properties of the most catalytically active sites of the particles. Typically, these sites are closely related to the structural defects at the surface of the particle (edges, vertices, etc.), and are presented by low-coordinated atoms. This fact gives us a possibility to relate calculations for small particles to experiments performed on larger particles that still contain the same types of the defect sites.

The calculations were based on the density-functional theory (DFT) with generalized gradient approximation (GGA) for exchange and correlation, and plane waves [15]. We used the GGA of Perdew, Burke, and Ernzerhof (PBE) [16], which gives good results for chemisorption of molecules at transition-metal surfaces. Ultrasoft scalar relativistic pseudopotentials and the VASP code [17] were used. The energy cutoff for the plane-wave basis was set at $400 \mathrm{eV}$, and all integrations over the Brillouin zone were done using the Monkhorst-Pack scheme with one $\mathbf{k}$ point in the relevant irreducible wedge [18]. Inclusion of additional $\mathbf{k}$ points was found to have minimal effect on the total-energy differences of interest here. Most of the calculations were spinpolarized because the magnetic ordering in small transition metal particles is important and significantly contributes in their total energies. However, we performed test non-spin-polarized calculations for $\mathrm{O}, \mathrm{S}$, and $\mathrm{SO}_{n}(n=1,2,3)$ species adsorbed on the nanoparticles and found that the reaction energies and activation barriers of interest were not significantly affected by magnetic ordering in the particles. Adsorption energies for $\mathrm{O}, \mathrm{S}$, and $\mathrm{SO}_{n}$ species at various sites on the nanoparticles, including "perimeter" sites, were calculated by optimizing local geometries. The minimum reaction paths and reaction barriers for the $\mathrm{SO}_{n}$ decomposition reactions were found using the nudged-elasticband method [19]. We did not perform any finite temperature modeling (such as first-principles molecular dynamics simulations) here. The total energies for different configurations were calculated and compared at zero degrees Kelvin. The finite temperature effects were introduced by using the calculated values of the reaction barriers for estimation of the reaction rates for different chemical processes.

\section{Results}

\subsection{Experimental results}

Sulfur dioxide production rates for the fresh PGM catalysts are shown in Fig. 2. It should be noted that these apparent reaction rates are qualitative in nature. Whereas the acid conversions were low ( $<5 \mathrm{~mol} \%$ ) for the Rh, Ir, and Ru catalysts, at the same catalyst loading, the conversion over the Pt catalyst approached about $1 / 2$ of the equilibrium limited value. Additionally, metal particle sizes, surface areas, and morphologies were not characterized for the various samples. However, the results suggest that the platinum catalyst had the highest activity, followed by the palladium catalyst. Production rates over the Rh, Ir, and Ru catalysts appeared to be substantially lower than over the Pt catalyst. The Ir and Ru catalysts displayed similar reaction rates which appeared to be lower than that measured over the Rh catalyst. The reaction was also studied over titania (rutile) without metal, but production rates were about five times lower than that measured over Ir. One other item of note is that the $\mathrm{SO}_{2}$ production rate over the $\mathrm{Pd}$ and $\mathrm{Pt}$ catalysts declined very rapidly, where as the production rates over the three harder metal catalysts were relatively stable (not shown).

\subsection{The $\mathrm{SO}_{3}$ decomposition mechanisms}

The results discussed here and in Section 3.3 are based on the computational study. As we mentioned above, at temperatures above $350^{\circ} \mathrm{C}$, a non-catalytic thermal decomposition of the sulfuric acid to gaseous $\mathrm{SO}_{3}$ and $\mathrm{H}_{2} \mathrm{O}$ takes place. Therefore, when modeling the catalytic decomposition of $\mathrm{SO}_{3}$ at temperatures above $750 \mathrm{C}$ one may consider an interaction between an individual $\mathrm{SO}_{3}$ molecule and the catalyst (metal particles on an oxide substrate).

First, we found that the catalytic activity of a pure oxide substrate is very low for both the alumina and titania substrates (in all the considered phases) which is in good agreement with our experimental results that show very low catalytic activity for both the pure $\mathrm{TiO}_{2}$ and $\mathrm{Al}_{2} \mathrm{O}_{3}$ surfaces. Calculations show that all the processes in which a single oxygen atom detaches from an $\mathrm{SO}_{3}$

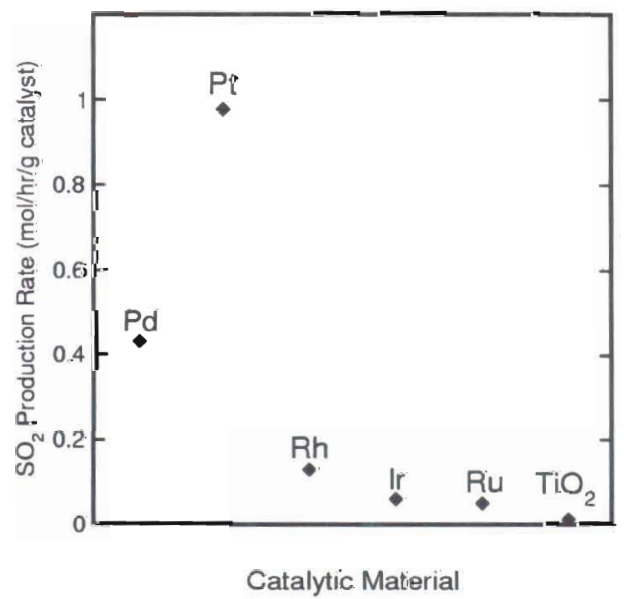

Fig. 2. Experimental $\mathrm{SO}_{2}$ production rates over various fresh platinum group metals supported on titania (rutile) at $8500^{\circ} \mathrm{C}$, a WHSV $=230 \mathrm{~g} \mathrm{H}_{2} \mathrm{SO}_{4} / \mathrm{g}$ catalyst/h, and total catalytic metal loadings of $0.1 \mathrm{wt} \%$. The experimental $\mathrm{SO}_{2}$ production rate for a pure $\mathrm{TiO}_{2}$ is also shown. 
molecule and attaches to an oxide surface is highly endothermic (the total energy of the system is several eVs higher after the reaction than before), i.e., the decomposition of $\mathrm{SO}_{3}$ at pure oxide surfaces is energetically very unfavorable (mainly because of the need to break a strong $\mathrm{S}-\mathrm{O}$ bond). Even in the presence of oxygen vacancies at the titania surface (which are more attractive sites than a pure surface for retaining $\mathrm{O}$ atoms after they have left the $\mathrm{SO}_{3}$ molecules), an additional $1.5-2 \mathrm{eV}$ is needed to tear an oxygen atom apart from an $\mathrm{SO}_{3}$ molecule.

When metal particles are deposited at an oxide surface, the situation may change especially when these particles contain lowcoordination reactive sites (located at the corners, edges, etc.). These sites may bind the reaction products $\left(\mathrm{SO}_{2}\right.$ and $\left.\mathrm{O}\right)$ stronger than a pure oxide would, which makes the decomposition reaction exothermic. The binding energies depend on the coordination number of the site and the type of the metal atoms in the particle. In this work we performed calculations for the PGM (Pd, Pt, Rh, Ir, $\mathrm{Ru}, \mathrm{Os}$ ) metals which have high melting temperature, $T_{\mathrm{m}}$, and high sublimation enthalpy, $\Delta H_{\mathrm{s}}$ (Table 1 ; actually, these two parameters are closely related, in particular, the ratio $\Delta H_{\mathrm{s}} / k T_{\mathrm{m}}$ is nearly a constant for all of the PGMs [20]).

The decomposition process starts with attaching an individual $\mathrm{SO}_{3}$ molecule to the metal particle (Fig. 3). Most likely, the molecule will initially attach to the cluster by a single atom ( $\mathrm{S}$ or $\mathrm{O}$ ). The binding energy is higher for the S-attachment configuration (Fig. 3b). Table 1 shows the $\mathrm{SO}_{3}$ binding energies to the $3-$ coordinated sites for different PGM particles. In general, there is an apparent correlation between the bulk melting temperature of the metals and the $\mathrm{SO}_{3}$ binding energies to the particle made of this metal, i.e., "harder" metal particles (i.e., particles made of metals with higher sublimation enthalpy) bind $\mathrm{SO}_{3}$ molecules stronger than "softer" ones. Another interesting observation is that the binding energies are higher than the barriers for tilting and rotation of the molecule as a whole around the attached atomthese barriers are not higher than $0.4 \mathrm{eV}$ for all of the considered metal particles. This means that the attached molecule will perform these motions before another atom of the molecule gets close enough to another site of the particle and attaches to it.

Further behavior of the $\mathrm{SO}_{3}$ molecule depends on the relation between the $\mathrm{S}-\mathrm{O}$ bond breaking reaction barrier (Fig. 4 ) and the change (positive or negative) of the total energy of the system due to such a breaking. Table 1 shows that for all of the considered metals, the change of the total energy is negative (the reaction is exothermic), and the $\mathrm{S}-\mathrm{O}$ bond breaking barrier is below $1.1 \mathrm{eV}$, i.e., the $\mathrm{SO}_{3}$ decomposition on small metal particle is very likely at high temperatures. Also, it is likely that the $\mathrm{SO}_{3}$ decomposition process will not stop here-the attached $\mathrm{SO}_{2}$ molecule (a product of the $\mathrm{SO}_{3}$ decomposition) may decompose into ( $\left.\mathrm{SO}+\mathrm{O}\right)$, and $\mathrm{SO}$ may

Table 1

The melting temperature $\left(T_{\mathrm{m}}\right)$ and the sublimation enthalpy [20] $\left(\Delta H_{\mathrm{s}}\right)$ of the considered PGMs as well as the energy parameters for the $\mathrm{SO}_{3}$ decomposition on the 3-coordinated site of the PGM particle positioned on rutile and $\gamma$-alumina surfaces: $E_{\mathrm{B}, \mathrm{S}}$ and $E_{\mathrm{B}, \mathrm{O}}$ are the binding energies of $\mathrm{SO}_{3}$ molecule attached to the 3-coordinated site by a single $\mathrm{S}$ or $\mathrm{O}$ atom; $\mathrm{E}_{\mathrm{barr}}$ is the reaction barrier for the $\mathrm{SO}_{3} \rightarrow \mathrm{SO}_{2}+\mathrm{O}$ decomposition process; $\Delta E_{\mathrm{tot}}$ is the change of the total energy of the system (the difference between the total energies of the supercell after and before the reaction)

\begin{tabular}{lllllll}
\hline Metal & Pd & Pt & Rh & Ir & Ru & Os \\
\hline$T_{\mathrm{m}}\left({ }^{\circ} \mathrm{C}\right)$ & 1559 & 1768 & 1964 & 2466 & 2334 & 3033 \\
$\Delta H_{\mathrm{s}}(\mathrm{eV})$ & 3.931 & 5.831 & 5.756 & 6.964 & 6.777 & 8.175 \\
$E_{\mathrm{B}, \mathrm{S}}(\mathrm{eV})$ & $-1.2 /-1.2$ & $-1.7 /-1.5$ & $-1.8 /-1.7$ & $-2.1 /-2.2$ & $-2.5 /-2.3$ & $-3.0 /-2.9$ \\
$E_{\mathrm{B}, \mathrm{O}}(\mathrm{eV})$ & $-0.7 /-0.8$ & $-0.9 /-1.0$ & $-1.1 /-1.3$ & $-1.7 /-1.9$ & $-2.0 /-1.8$ & $-2.4 /-2.5$ \\
$E_{\text {barr }}(\mathrm{eV})$ & $1.1 / 1.0$ & $0.9 / 0.7$ & $0.7 / 0.6$ & $0.6 / 0.6$ & $0.3 / 0.4$ & $0.5 / 0.4$ \\
$\Delta E_{\text {tot }}(\mathrm{eV})$ & $-0.7 /-0.4$ & $-0.5 /-0.6$ & $-1.0 /-1.2$ & $-1.8 /-1.6$ & $-1.8 /-1.9$ & $-2.4 /-2.3$ \\
\hline
\end{tabular}

The energy parameters for the rutile/ $\gamma$-alumina surfaces are shown above/below the "/" sign.
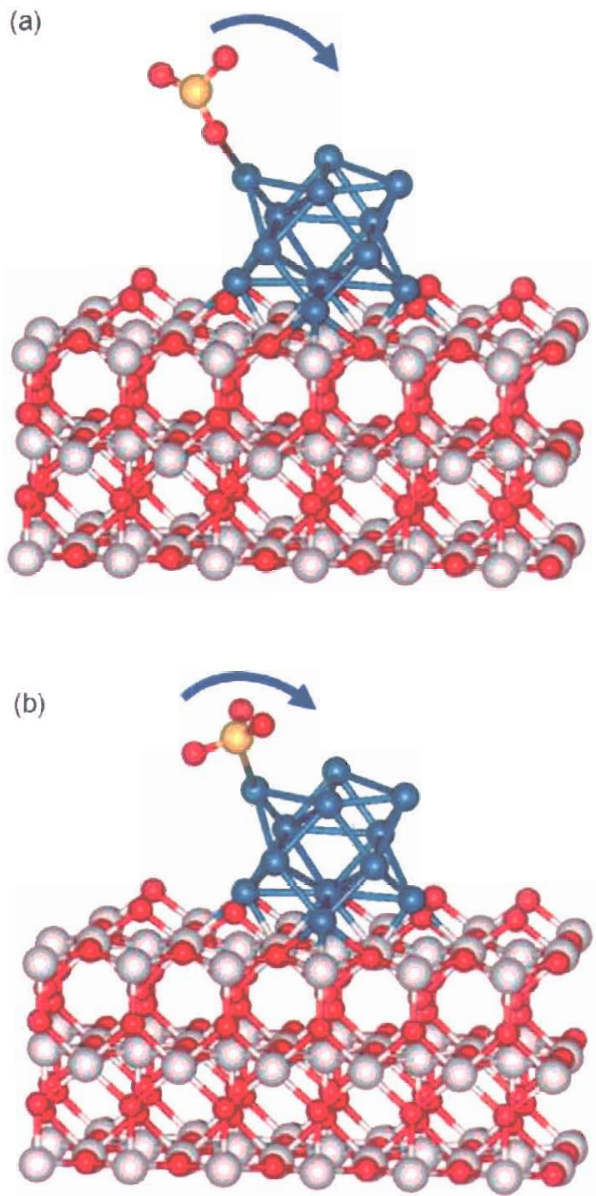

Fig. 3. Two binding configurations for an attached single $\mathrm{SO}_{3}$ molecule to a 3 coordinated Pt site on a 12 -atoms Pt nanoparticle positioned on the rutile surface. Ti atoms are shown in light grey, $\mathrm{O}$ in red, $\mathrm{Pt}$ in blue, $\mathrm{S}$ in yellow. $\mathrm{SO}_{3}$ may be attached by an $\mathrm{O}$ atom (a) or by the $\mathrm{S}$ atom (b). The arrows show some possible directions of the rotation and tilting motions of the attached molecule as a whole. (For interpretation of the references to color in this figure legend, the reader is referred to the web version of the article.)

decompose into $(\mathrm{S}+\mathrm{O})$. Therefore, one may expect that $\mathrm{SO}_{n}$ species with different oxygen content will be formed on the metal particles. It should also be worth mentioning that we did not find any significant differences between similar particles positioned on $\mathrm{TiO}_{2}$ or $\mathrm{Al}_{2} \mathrm{O}_{3}$ substrates - the shape and composition of the particle is more important than the type of the oxide surface on which the particle has been deposited. An interesting observation is that the binding energy of a molecule to the nanoparticle is lowering (Fig. 5) and the $\mathrm{SO}_{3}$ decomposition barrier is increasing (Fig. 6) with the number of d-electrons for metals within the same transition metal period ( $4 \mathrm{~d}$ or $5 \mathrm{~d}$ ). These results may assist in making predictions for catalytic properties of particles made of PGM alloys.

Here we performed calculations for small TM particles which are highly reactive because they contain a large number of lowcoordinated (3-and 4-coordinated) TM sites that strongly bind the reaction products thus making the whole $\mathrm{SO}_{3}$ decomposition process exothermic. On larger particles, the decomposition still occurs on the "defects", i.e., on the low-coordinated sites positioned at the vertices and edges of the particle. However, the process becomes less energetically favorable because (i) the relative number (per metal atom) of the low-coordinated sites decreases, and (ii) the binding energies of the decomposition products to the high-coordinated sites are lower than to the low- 

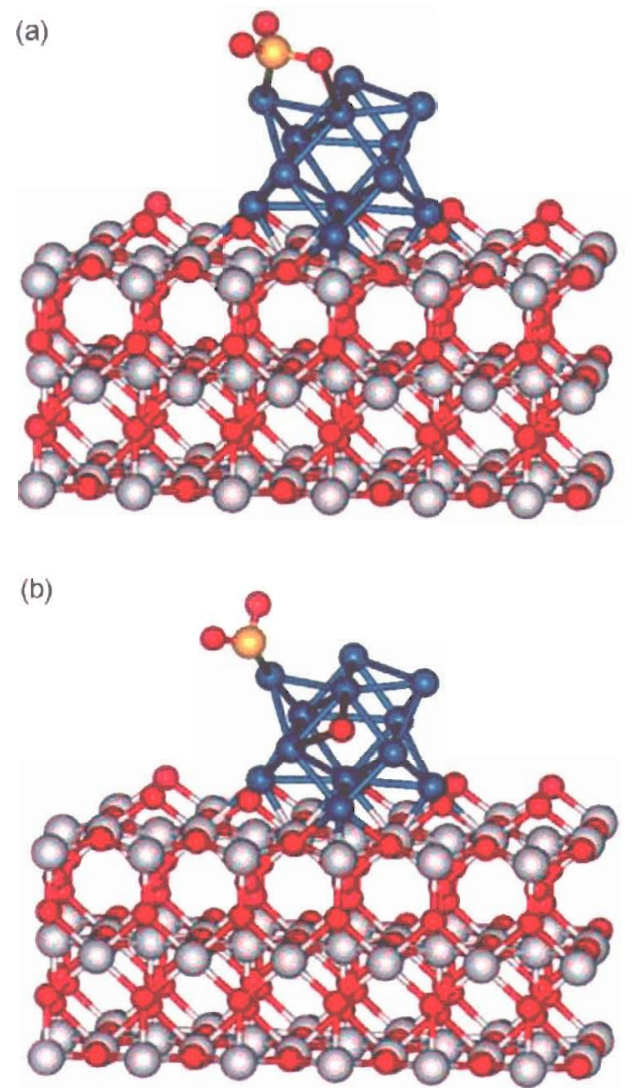

Fig. 4. $\mathrm{SO}_{3}$ decomposition on a 12-atoms $\mathrm{Pt}$ nanoparticle on the rutile surface Schematics of the atomic configurations before (a) and after (b) the decomposition.

coordinated sites. Moreover, for flat surfaces of all of the considered bulk metals, the $\mathrm{SO}_{3}$ decomposition is endothermic, with an additional energy (between 1 and $2 \mathrm{eV}$ ) needed. This is one of the main reasons why the catalytic activity of the particles decreases with their growth and sintering. Here we did not perform a detailed statistical analysis of the $\mathrm{SO}_{3}$ decomposition rate as a function of the size and shape of the particles (similar to the analysis performed for gold nanoparticles [21]). The statistical analysis of the $\mathrm{SO}_{3}$ decomposition will be discussed elsewhere.

\subsection{Removal of the decomposition products from nanoparticles}

In the previous subsection we showed that small PGM's nanoparticles are quite efficient in assisting the $\mathrm{SO}_{3}$ decomposition. An active catalyst, however, should be free of poisoning by the reaction products. This means that there should exist some efficient mechanisms for removing atomic $\mathrm{O}, \mathrm{S}$, as well as $\mathrm{SO}_{n}$ $(n=1,2)$ species from a particle after a $\mathrm{SO}_{3}$ molecule decomposes on this particle.

First, we analyze mechanisms of the atomic oxygen removal from a particle. Obviously, the efficiency of this process depends on the binding energy of the $\mathrm{O}$ atom to the particle, i.e., on the coordination of the particle site(s) to which the $\mathrm{O}$ atom is attached (in different atomic configurations, $\mathrm{O}$ may attach to one, two, or three metal atoms). When the particle is large and may be simulated by a flat bulk metal surface (Fig. 7), the results have no variations because all the surface sites are equivalent. In spite of the fact that $\mathrm{O}$ binds to the "bulk" PGM surfaces quite strong (the binding energy varies between $(-3.2 \mathrm{eV})$ for the Pd (1 111$)$ and $(-5.6 \mathrm{eV})$ for the Os $\left(\begin{array}{llll}0 & 0 & 0 & 1\end{array}\right)$ surfaces, Table 2$)$, the surface oxygen

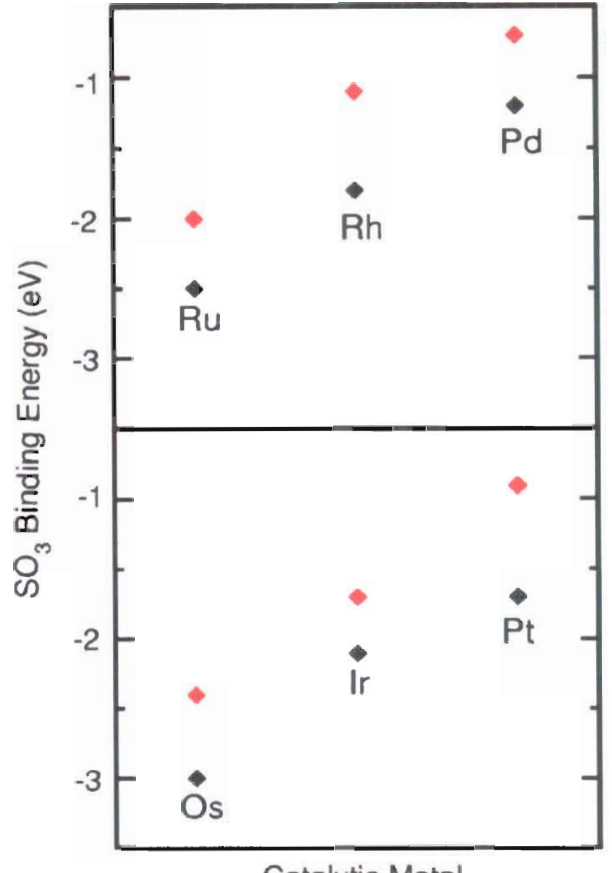

Catalytic Metal

Fig. 5. The binding energies of $\mathrm{SO}_{3}$ molecule attached to the 3-coordinated site of a PGM cluster anchored on the $\mathrm{TiO}_{2}$ substrate by a single $\mathrm{S}\left(E_{\mathrm{B}, \mathrm{S}}\right.$, black diamonds) or $\mathrm{O}$ ( $E_{\mathrm{B}, 0}$, red diamonds) atom for all of the considered PGMs (see also Table 1). The energy parameters for the $4 \mathrm{~d}$ (5d) PGM metals are shown in the upper (lower) panel. (For interpretation of the references to color in this figure legend, the reader is referred to the web version of the article.)

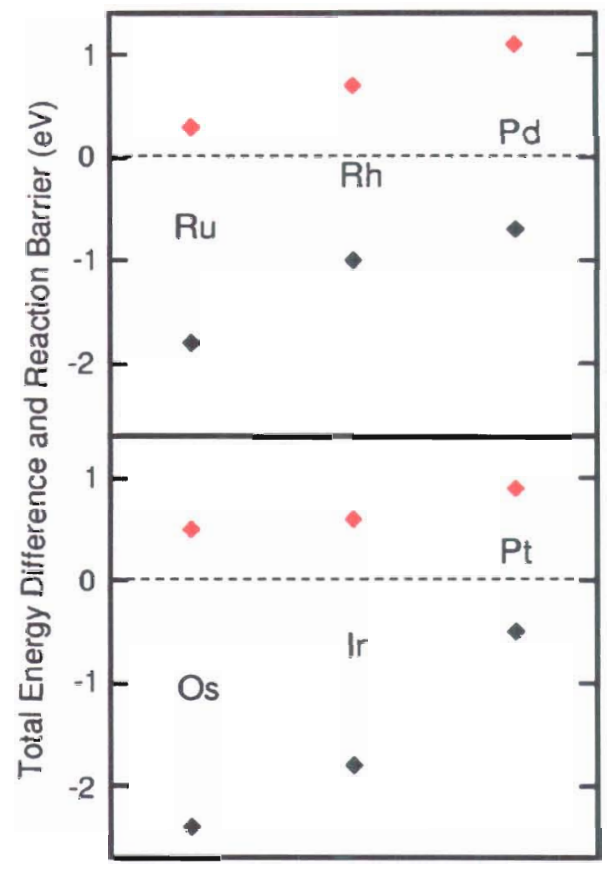

Catalytic Metal

Fig. 6. The reaction barrier for the $\mathrm{SO}_{3} \rightarrow \mathrm{SO}_{2}+\mathrm{O}$ decomposition process ( $E_{\mathrm{bar}}$, red diamonds) and the change of the total energy of the system (the difference between the total energies of the supercell after and before the reaction $-\Delta E_{\text {tot }}$, black diamonds) for the decomposition reaction on a PGM cluster positioned on rutile (see also Table 1). The energy parameters for the 4d (5d) PGM metals are shown in the upper (lower) panel. (For interpretation of the references to color in this figure legend, the reader is referred to the web version of the article.) 


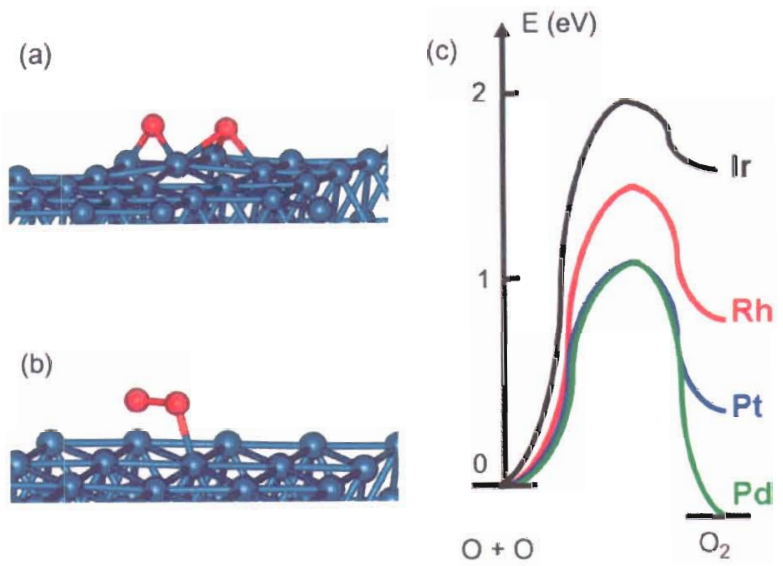

Fig. 7. (a) Pd ( $\left.\begin{array}{lll}1 & 1 & 1\end{array}\right)$ surface with two attached $\mathrm{O}$ atoms positioned in the neighboring surface sites. $\mathrm{Pd}$ atoms are shown in blue, $\mathrm{O}$ in red; (b) formation of an $\mathrm{O}_{2}$ molecule that is still bound to the surface; (c) a schematic showing the $\mathrm{O}_{2}$ formation reaction path and reaction barrier as well as the total energy gain for the "bulk" surfaces of the four PGMs with the fcc structure. (For interpretation of the references to color in this figure legend, the reader is referred to the web version of the article.)

migration barriers are quite low and vary between 0.3 and $0.5 \mathrm{eV}$ for all of the considered metals. Therefore, it seems unlikely that oxygen leaves the surface in an atomic form. Instead, $O$ atoms have a tendency to migrate at the surface and interact with each other. The question about the possibility of forming an $\mathrm{O}_{2}$ molecule should be analyzed for each of the considered metal surfaces individually. Fig. $7 \mathrm{c}$ shows the $\mathrm{O}_{2}$ formation barriers for the four PGMs that crystallize in the fcc structure-Pd, Pt, Rh, and Ir. The formation of $\mathrm{O}_{2}$ is energetically favorable only for Pd and is slightly unfavorable for Pt while for Rh and Ir (and for the two hcp metals Ru and Os, see Table 2) such a formation is very unlikely. Also, the barrier of the reverse process (decomposition of $\mathrm{O}_{2}$ on atoms, $E_{\mathrm{barr}}$ $\left(\mathrm{O}_{2} \rightarrow 2 \mathrm{O}\right)$ ) is lower for these metals than for Pt and Pd, while the binding energy of $\mathrm{O}_{2}$ to the surfaces of these metals, $E_{\mathrm{B}}^{\mathrm{O}_{2}}$, is higher than $2.3 \mathrm{eV}$. This means that oxygen removal from the surface by the formation of $\mathrm{O}_{2}$ should be much slower for $\mathrm{Rh}, \mathrm{Ir}, \mathrm{Ru}$, and $\mathrm{Os}$ than for Pd and Pt hecause (i) the $\mathrm{O}_{2}$ formation on the surfaces of

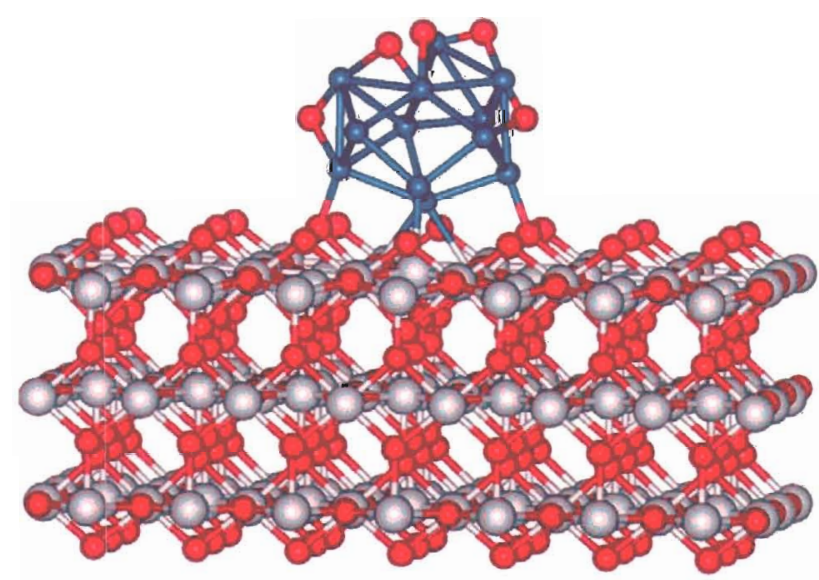

Fig. 8. A configuration of five oxygen atoms attached to a 12-atoms Ir (shown in blue) nanoparticle positioned on the rutile surface. The binding energy of each 0 atom to the particle is practically independent on a presence of other neighboring $O$ atoms. (For interpretation of the references to color in this figure legend, the reader is referred to the web version of the article.)
Table 2

The energy parameters characterizing the behavior of atomic and molecular oxygen at the "bulk" PGM surfaces (with the $\left(\begin{array}{lll}1 & 1 & 1\end{array}\right)$ orientation for the fcc metals Pd, Pt, Rh, and $\mathrm{Ir}$, and $\left(\begin{array}{llll}0 & 0 & 0 & 1\end{array}\right)$ orientation for the hcp metals Ru and $\left.O s\right): E_{\mathrm{B}}^{\mathrm{O}}$ is the binding energy of an $O$ atom to the surface; $E_{\text {migr }}^{0}$ is the migration barrier for atomic oxygen at the surface: $E_{\text {barr }}\left(2 \mathrm{O} \rightarrow \mathrm{O}_{2}\right)$ is the barrier of the formation of an $\mathrm{O}_{2}$ molecule at the surface; $E_{\mathrm{tot}}^{\mathrm{O}}-E_{\mathrm{tot}}^{2 \mathrm{O}}$ is the difference between the total energy of the supercells with $\mathrm{O}_{2}$ and the system with two $\mathrm{O}$ atoms; $E_{\text {barr }}\left(\mathrm{O}_{2} \rightarrow 2 \mathrm{O}\right)$ is the barrier of the $\mathrm{O}_{2}$ decomposition at the surface; $E_{\mathrm{B}}^{\mathrm{O}_{2}}$ is the binding energy of $\mathrm{O}_{2}$ to the surface

\begin{tabular}{lrrrrrr}
\hline Metal & \multicolumn{1}{l}{ Pd } & \multicolumn{1}{l}{ Pt } & \multicolumn{1}{l}{ Rh } & \multicolumn{1}{c}{ Ir } & \multicolumn{1}{c}{ Ru } & Os \\
\hline$E_{\mathrm{B}}^{\mathrm{O}}(\mathrm{eV})$ & -3.2 & -3.6 & -4.1 & -4.7 & -4.9 & -5.6 \\
$E_{\mathrm{migr}}^{\mathrm{O}}(\mathrm{eV})$ & 0.5 & 0.5 & 0.4 & 0.3 & 0.4 & 0.3 \\
$E_{\text {barr }}\left(2 \mathrm{O} \rightarrow \mathrm{O}_{2}\right)(\mathrm{eV})$ & 1.1 & 1.2 & 1.4 & 1.9 & 2.1 & 2.4 \\
$\left(E_{\mathrm{tot}}^{\mathrm{O}_{2}}-E_{\text {tot }}^{2 \mathrm{O}}\right)(\mathrm{eV})$ & -0.2 & +0.3 & +0.7 & +1.6 & +1.9 & +2.2 \\
$E_{\text {barr }}\left(\mathrm{O}_{2} \rightarrow 2 \mathrm{O}\right)(\mathrm{eV})$ & 1.3 & 0.9 & 0.7 & 0.3 & 0.2 & 0.2 \\
$E_{\mathrm{B}}^{\mathrm{O}_{2}}(\mathrm{eV})$ & -1.4 & -1.8 & -2.3 & -2.7 & -2.9 & -3.8 \\
\hline
\end{tabular}

these metals is very unlikely: (ii) even if $\mathrm{O}_{2}$ is formed, it binds strongly to the surface, and it takes a longer time to be removed.

The $O$ binding energies to small particles (with lower coordination of metal atoms at their surfaces) are stronger than to flat surfaces, i.e., small particles have even higher tendency to hold $\mathrm{O}$ atoms than large particles (Fig. 8). Although the $\mathrm{O}$ atoms still have a possibility to migrate around the surface of the particle (for a 12-atom Ir particle shown in Fig. 8, the $\mathrm{O}$-migration barrier is about $0.8 \mathrm{eV}$ only), the $\mathrm{O}_{2}$ formation process is very endothermic for small particles, and therefore, the most active sites of the particle have a tendency to hold atomic oxygen (one of the reaction product) for a long time on an atomic site.

Before now, we consider only individual oxygen atoms and molecules at metal surfaces. It is known, however, that for flat surfaces of some metals (e.g., Pt) the binding energy of oxygen to the surface declines with the surface coverage before reaching saturated values at a steady-state regime (See Refs. [22-24] and references therein). Another interesting phenomenon is an incorporation of oxygen into the metal at the Ru ( 00001$)$ surface, with a formation of oxygen-rich subsurface islands which characterizes an onset of the oxide film formation [25]. The binding energy of the adsorbed surface oxygen atoms also decreases while the subsurface islands are formed underneath. We would also expect that the oxygen binding energies will decline with coverage for all of the considered metals and a steadystate regime will be reached for any values of the external parameters, such as temperature, pressure, etc. A complete statistical analysis of these effects goes beyond the scope of this publication. However, we expect (comparing the values of the binding energies for the atomic and molecular oxygen in Table 2) that the steady-state oxygen coverage is higher for the "harder" metals (Rh, Ir, Ru, and Os) than for the "softer" metals (Pd and Pt). Also, for small particles the dependence of the binding energy of oxygen to the surface on the oxygen coverage is weaker than for flat surfaces, i.e., the binding energy for each added oxygen atom (especially to the low-coordinated sites) is nearly independent on the number of $\mathrm{O}$ atoms located at the particle.

In addition to the presence of oxygen that binds to the most active sites at the nanoparticles thus reducing its activity, a formation of $\mathrm{SO}_{n}$ species at the particles is also possible. The mechanism of the "sulfate" poisoning can be understood from an example shown in Fig. 9 that shows an SO group attached to a 3coordinated site of a Pt cluster by the $\mathrm{S}$ atom. The energy barrier to break the $\mathrm{Pt}-\mathrm{S}$ bond is quite high $(\sim 3 \mathrm{eV})$, i.e., such a process would not occur even at temperatures used for the $\mathrm{SO}_{3}$ decomposition process. However, the SO group can easily bend (the bending barrier is $0.3 \mathrm{eV}$ only, Fig. 9a) until the $\mathrm{O}$ atom also attaches to the particle and subsequently the SO bond breaks (the 

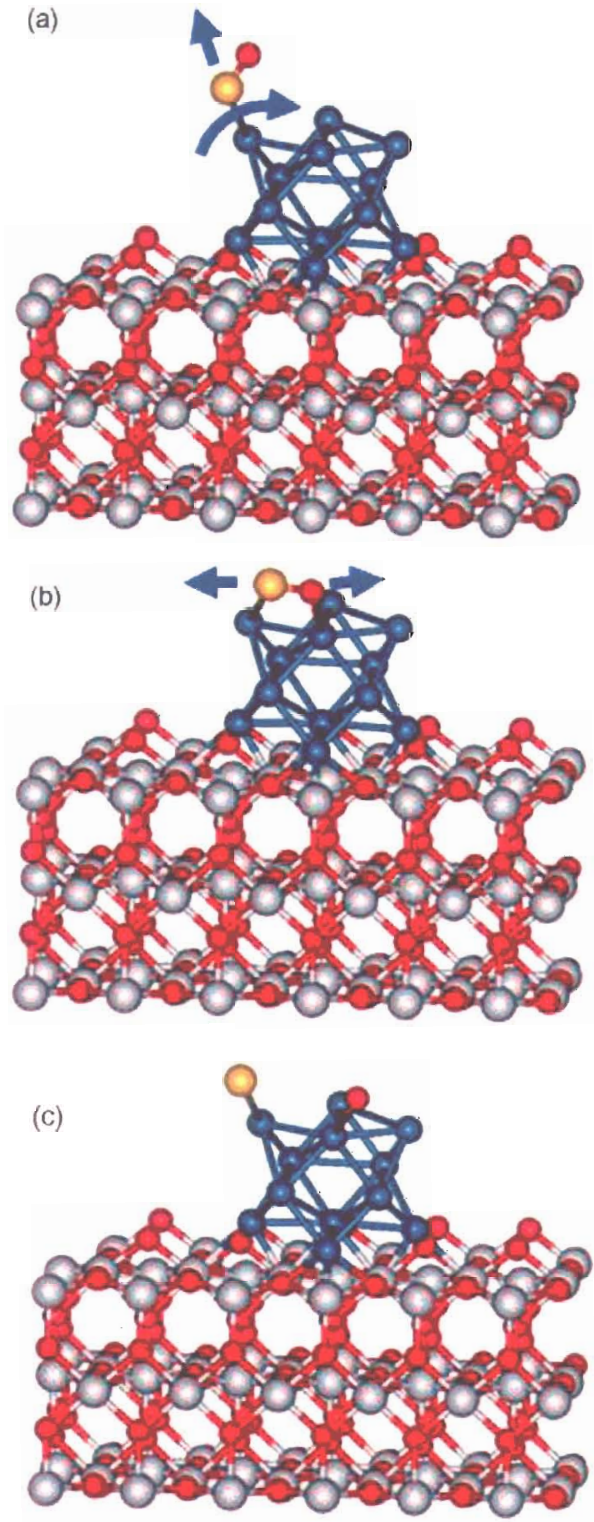

Fig. 9. Possible transformations of an SO group attached to a Pt nanoparticle. (a) Attachment of the SO group by the $\mathrm{S}$ atom. The arrows show the two possible motions of the group as a whole (detaching from the cluster or bending before the 0 atom also attaches); (b) breaking of the SO bond with a formation of the two individual ( $\mathrm{S}$ and $\mathrm{O}$ ) atoms positioned on the particle $(\mathrm{c}$ )

breaking barrier is about $1 \mathrm{eV}$, Fig. 9b). After these two transformations, one gets a group of two individual atoms ( $S$ and $\mathrm{O}$ ) positioned at the particle (Fig. 9c). In one case, these two atoms may reconnect back and form the SO group again. in another case, the two individual $\mathrm{S}$ and $\mathrm{O}$ atoms may start to migrate individually around the surface of the particle. An important fact is that in both cases $\mathrm{S}$ and $\mathrm{O}$ atoms remain on the particle (connected or disconnected), and in both cases, the energy barrier to move them away is quite high. When additional $\mathrm{S}$ and $\mathrm{O}$ atoms are present at the surface, a formation of different $\mathrm{SO}_{n}$ species and their aggregates is possible. These structures can also block catalytically active sites at the surface thus reducing the catalytic activity of the system. Calculations show that such a mechanism of the $\mathrm{SO}_{n}$ formation may be very efficient for all of the considered PGMs.
The example considered above was just an illustration of how the $\mathrm{SO}_{n}$ groups can initiate the formation of "sulfates" at the surface of the particle. The binding energy of these species to the particle may vary in a wide range depending on the coordination of the site to which the group is attached. The value of $3 \mathrm{eV}$ taken above for an $\mathrm{SO}$ group is the maximal value of the binding energy of SO to a Pt particle (to the 3-coordinated site). On the other sites of the same particle this energy may be as low as $1.2 \mathrm{eV}$. The same thing is true for the attached $\mathrm{SO}_{2}$ and $\mathrm{SO}_{3}$ molecules also. Therefore, not all of the reacting $\mathrm{SO}_{n}$ species will contribute into the surface poisoning. Another important factor is the dynamical rearrangements of different "sulfate" species at the surface. In particular, the traveling $\mathrm{S}$ and $\mathrm{O}$ atoms may reconnect at another particle site which does not bind the SO group as strong as the lowcoordinated site on which the SO group was initially decomposed on individual atoms. Therefore, the problem of poisoning should be investigated statistically which is beyond the scope of the present work.

\section{Discussion}

Our calculations suggest that the catalytic activity of the oxidesupported PGM nanoparticles is defined in large part by (i) the energy barrier for the detachment of $\mathrm{O}$ atoms from the $\mathrm{SO}_{n}(n=1,2$, 3 ) species, and (ii) the removal rate of the products of the sulfuric acid decomposition (atomic $\mathrm{O}, \mathrm{S}$, and the $\mathrm{SO}_{n}$ species) from nanoparticles. Basically, all the PGM (Pd, Pt, Rh, Ir, Ru, and Os) nanoparticles are efficient in assisting the decomposition of $\mathrm{SO}_{3}$ because of their ability to bind the reaction products strongly thus making the whole process exothermic. Also, the decomposition barriers for $\mathrm{SO}_{3}$ attached to small nanoparticle are quite low (varying between $0.3 \mathrm{eV}$ for $\mathrm{Ru}$ and $1.1 \mathrm{eV}$ for $\mathrm{Pd}$ on a rutile substrate, Table 1 ). Therefore, for the case of a clean catalyst, the $\mathrm{SO}_{3}$ decomposition rate at the temperatures above $750{ }^{\circ} \mathrm{C}$ will be quite high. Moreover, it will be higher on the "harder" metal ( $\mathrm{Rh}, \mathrm{Ir}$, $\mathrm{Ru}$, and $\mathrm{Os}$ ) than on the "softer" metal (Pd and Pt) particles. The type of the oxide substrate (titania vs. alumina) as well as other details of the substrate structure (e.g., a presence of oxygen vacancies on a titania surface) are not significant. The presence of defect sites (low-coordinated atoms) at the particle is more important. Only the particles that contain a significant fraction of low-coordinated atoms at their surfaces (edges, vertices, etc.) are efficient as catalysts for the $\mathrm{SO}_{3}$ decomposition. With the growth and sintering of the particles, the activity of the system continuously decreases as the number of low-coordinated surface is reduced.

However, the experimental sulfur dioxide production rates for the fresh PGM catalysts are higher for the "softer" metals (Pd, Pt) than for the "harder" metals (Rh, Ir, Ru, and Os, see Fig. 2). We attribute such a behavior to a much higher potential of the "harder" metals to strongly attach the $\mathrm{SO}_{3}$ decomposition products- $\mathrm{S}, \mathrm{O}, \mathrm{SO}$, and $\mathrm{SO}_{2}$. $\mathrm{S}$ and $\mathrm{O}$ individual atoms as well as the $\mathrm{SO}_{n}$ species migrate around the surface of the particle, bind to each other and form some structures on the top of the particle that block the most catalytically active sites. The "harder" PGMs are much less efficient in removing the reaction products from the nanoparticles than the "soft" PGMs (Table 2). The binding energies of the reaction products to the nanoparticles are much higher than the $\mathrm{SO}_{3}$ decomposition energy barriers on the particles. Therefore, the decomposition rate limiting step is mainly defined by the removing of oxygen and sulfates from the particles rather than to the $\mathrm{SO}_{3}$ decomposition on the particle.

Comparing the efficiency of the two "softer" metals (Pd and Pt) we see that for $\mathrm{Pd}$, the energy one needs to detach $\mathrm{SO}_{3}$ from the cluster $\left(-E_{\mathrm{B} . \mathrm{S}}=1.2 \mathrm{eV}\right)$ is approximately equal to the decomposi- 
tion barrier $E_{\text {barr }}=1.1 \mathrm{eV}$ while for Pt $\left(-E_{\mathrm{B}, \mathrm{S}}\right)$ is essentially higher than $E_{\text {barr }}$ (Table 1). This means that each $\mathrm{SO}_{3}$ molecule attached to the Pd cluster has a probability of about one half to decompose and about one half to detach and fly away while an $\mathrm{SO}_{3}$ attached to a similar Pt cluster is more likely to decompose. Therefore, the $\mathrm{SO}_{3}$ decomposition rate on a Pt cluster should be about twice higher than the rate on a Pd cluster, in a good agreement with Fig. 2.

An important conclusion here is that the $\mathrm{SO}_{3}$ decomposition rate on a PGM particle is mainly defined by the removal rate of the reaction products from the particle. Such a poisoning effects (accumulation of the reaction products on the surface of the particle) is the strongest for sites that exhibit the highest catalytic activity. Therefore, the "softer" metal nanoparticles exhibit a higher catalytic activity than the "harder" ones.

\section{Conclusions}

In summary, we have presented an extensive theoretical study of the catalytic activity of PGM (Pd, Pt, Rh, Ir, Ru, and Os) nanoparticles positioned on four different oxide surfaces. The results for the catalytic activity and deactivation of these catalytic systems in the $\mathrm{SO}_{n}$ decomposition processes are in a good qualitative agreement with existing experimental data.

We found that there are several nanoscale features that collectively result in the observed behavior of these catalytic systems. We demonstrated that the same nanoscale feature such as a presence of low-coordinated sites on the metal cluster may play both positive (increasing the $\mathrm{SO}_{3}$ decomposition rate) and negative (increasing the poisoning) roles. Actually, for this reaction, it is important to have the right combination of lowand high-coordinated sites so the catalyst will be highly active and the reaction products will be able to desorb from the metal particles (by migrating to the high-coordinated sites) without rapidly poisoning the catalytic material. The $\mathrm{SO}_{3}$ decomposition rate on a particle is mainly defined by the removal rate of the reaction products from the particle. Therefore, at least one of the reasons that the particles made of "harder" metals ( $\mathrm{Rh}, \mathrm{Ir}, \mathrm{Ru}$, and Os), with the higher melting temperatures and sublimation enthalpy, experience lower catalytic activity because they bind the reaction products stronger than the "softer" metal (Pd and Pt) particles. In order to investigate the catalytic behavior of the system with time on a stream, one needs to take into account the stability of the PGM nanoparticles at high temperatures and their ability to grow and sinter. These factors will be considered in more details in a subsequent paper.

\section{Acknowledgments}

This work was supported through the INL Laboratory Directed Research and Development program and the U.S. Department of Energy, Office of Nuclear Energy under DOE Idaho Operations Office Contract DE-AC07-051D14517 and in part by the Division of Materials Sciences and Engineering of the Office of Basic Energy Sciences of the U.S. Department of Energy also under DOE Idaho Operation Office Contract DE-AC07-051D14517. This work was also supported in part by a grant of computer time from the DOE Office of Science National Energy Research Scientific Computer Center (NERSC).

\section{References}

[1] C.E. Bamberger, D.M. Richardson, Cryogenics 16 (1976) 197.

[2] D. O'Keefe, C. Allen, G. Besenbruch, L. Brown, J. Norman, R. Sharp, K. McCorkle, Int. J. Hydrogen Energy 7 (1982) 831.

[3] L.E. Brecher, S. Spewock, C.J. Warde, Int. J. Hydrogen Energy 2 (1977) 7.

[4] G.E. Beghi, Int. J. Hydrogen Energy 11 (1986) 761.

[5] D.R. O'Keefe, J.H. Norman, D.G. Williamson, Catal. Rev.-Sci. Eng. 22 (1980) 325.

[6] J.H. Norman, K.J. Mysels, R. Sharp, D. Williamson, Int. J. Hydrogen Energy 7 (1982) 545.

[7] H. Tagawa, T. Endo, Int. J. Hydrogen Energy 14 (1989) 11.

[8] High-Pressure Catalytic Metal Reactor in a Simulated Solar Central Receiver, GA Technologies Report GA-A18285, February 1986.

[9] D.M. Ginosar, A.W. Glenn, L.M. Petkovic, K.C. Burch, Int. J. Hydrogen Energy 32 (2007) 482.

[10] L.M. Petkovic, D.M. Ginosar, H.W. Rollins, K.C. Burch, P.J. Pinhero, H.H. Farrell, Appl. Catal. A: Gen. 338 (2008) 27.

[11] D.M. Ginosar, H.W. Rollins, L.M. Petkovic, K.C. Burch, M.J. Rush, High-temperature sulfuric acid decomposition over complex metal oxide catalysts, Int. J. Hydrogen Energy, submitted for publication.

[12] K. Sohlberg, S.J. Pennycook, S.T. Pantelides, J. Am. Chem. Soc. 121 (1999) 7493.

[13] H. Knozinger, P. Ratnasamy, Catal. Rev.-Sci. Eng. 17 (1978) 31.

[14] E. Wahlstrom, N. Lopez, R. Schaub, P. Thostrup, A. Rønnau, C. Africh, E. Lægsgaard, J.K. Nørskov, F. Besenbacher, Phys. Rev. Lett. 90 (2003) 026101.

[15] M.C. Payne, M.P. Teter, D.C. Allan, T.A. Arias, J.D. Joannopoulos, Rev. Mod. Phys. 64 (1992) 1045

[16] J.P. Perdew, K. Burke, M. Ernzerhof, Phys. Rev. Lett. 77 (1996) 3865

[17] G. Kresse, J. Hafner, Phys. Rev. B 48 (1993) 13115; G. Kresse, J. Furthmüller, Phys. Rev. B 54 (1996) 11169.

[18] D.J. Chadi, M.L. Cohen, Phys. Rev. B 8 (1973) 5747.

[19] H. Johnson, G. Mills, K.W. Jacobsen, in: B.J. Berne, G. Cicotti, D.F. Coker (Eds.), Classical and Quantum Dynamics in Condensed Phase Systems, World Scientific, River Edge, NJ, 1998.

[20] D.R. Linde (Ed.), CRC Handbook of Chemistry and Physics, 76th edition, CRC Press, Boca Raton, FL, 1995-1996, pp. 4-122.

[21] S.N. Rashkeev, A.R. Lupini, S.H. Overbury, S.J. Pennycook, S.T. Pantelides, Phys. Rev. B 76 (2007) 035438.

[22] A. Eichler, J. Hafner, Phys. Rev. B 59 (1999) 5960

[23] A. Alavi, P.J. Hu, T. Deutsch, P.L. Silvestrelli, J. Hutter, Phys. Rev. Lett. 80 (1998) 3650 .

[24] Y.Y. Yeo, L. Vattuone, D.A. King, J. Chem. Phys. 106 (1997) 392.

[25] K. Reuter, C. Stampfl, M.V. Ganduglia-Pirovano, M. Scheffler, Chem. Phys, Lett. 352 (2002) 311. 